\title{
MS058.P03
}

\section{Random electron diffraction tomography for structure analysis of pharmaceuticals}

Stavros Nikolopoulos ${ }^{1}$, Athanasios S. Galanis ${ }^{1}$, Oriol Vallcorba ${ }^{2}$, Alex Eggeman ${ }^{3}$, Partha Pratim Das ${ }^{1}$, Jan Pieter Abrahams ${ }^{4}$, Edgar Rauch $^{5}$, Paul A. Midgley ${ }^{3}$, Mauro Gemmi ${ }^{6}$

${ }^{1}$ NanoMEGAS SPRL, Brussels, Belgium, ${ }^{2}$ Experiments Division - MSPD Beamline, ALBA, Barcelona, Spain, ${ }^{3}$ Department of Materials Science and Metallurgy, University of Cambridge, Cambridge, United Kingdom, ${ }^{4}$ University of Basel, Basel, Switzerland, ${ }^{5}$ SIMAP Laboratory, CNRS-Grenoble INP, Grenoble, Switzerland, ${ }^{6}$ Center for Nanotechnology Innovation at NEST, Istituto Italiano di Tecnologia, Pisa, Italy

E-mail: info@nanomegas.com

Electron diffraction (ED) in a transmission electron microscope (TEM) has a number of advantages compared to X-ray diffraction as it allows to sample individually very small crystals (50-500 $\mathrm{nm}$ ). To study pharmaceutical samples in the TEM, cooling the sample at LN2 temperatures and working under low dose is mandatory (critical dose should not exceed 50-100 e-/A2). A TEM based 3D diffraction tomography has been used to study pharmaceutical compounds [1] where a nanocrystal in tilted in small steps or continuously, in order to reconstruct the (3D) reciprocal volume. However, where very small crystals $(<10 \mathrm{~nm})$ or beam sensitive materials should be analyzed, 3D diffraction tomography technique can be of limited use. In this work, we present a novel approach to analyze beam sensitive materials. By scanning the beam in the TEM over a certain area (eg $5 \times 5$ microm2) we can collect a big number of ED patterns where we can select "randomly" good quality ED patterns (ED having a big number of reflections, without need to be perfectly zone axis oriented patterns). A collection of limited number of ED patterns (usually 10-15) may allow determine unit cell parameters and by measuring ED intensities to solve crystal structures. Scanning speed should be fast enough (50-100 frames/sec) to allow diffraction happen before nanocrystals may be damaged by the electron beam. In our experiments we have used a CM300 FEG and Libra 120 TEM microscopes equipped with ASTAR/Digistar (NanoMEGAS) scanning precession diffraction device. Diffraction data were collected using low temperature with scanning area of $5 \times 5$ microns, $50 \mathrm{~nm}$ step size and $20 \mathrm{~nm}$ beam size. We show that VDF (virtual dark field) of scanned areas reveal (fig.1) clearly $<10 \mathrm{~nm}$ sized resorcinol crystals on amorphous carbon background. Selecting 6 ED patterns from various diffracting crystallites allowed us to reconstruct the unit cell using EDIff software [2] with values of $a=10.05 \AA, b=9.195 \AA, c=5.615 \AA$ (Literature reported values $10.53 \AA, 9.53 \AA, 5.60 \AA$ ). In another case , from 15 selected "randomly oriented" ED patterns of aspirin nanocrystals use of EDIff software revealed a monoclinic unit cell of $12.26 \AA 6.72 \AA, 11.07 \AA$, beta= 95 degree ( Literature reported values $11.43 \AA, 6.59 \AA, 11.39 \AA$, beta = 95.68 degree). Reflections intensities from those 15 "random oriented" ED patterns have been collected and analyzed with TALP software [3]; the structure was solved by fixing the molecular model (rigid block). The conclusion is that even with ED data coming from limited number of patterns, is still possible to solve the structure provided the molecular model and the right conformation are known. Our results indicate that our "random tomography" approach in the TEM may help to analyze beam sensitive pharmaceutical compounds where their unit cell parameters and possibly their structure can be also revealed.

[1] van Genderen, E., Clabbers, M. T. B., Das, P. P., Stewart, A., Nederlof, I., Barentsen, K. C., Portillo, Q., Pannu, N. S., Nicolopoulos, S., Gruene, T. \& Abrahams, J. P. (2016). Acta Cryst. A72, 236-242.

[2] Jiang, L., Georgieva, D. \& Abrahams, J. P. (2011). J. Appl. Cryst. 44, 1132-1136

[3] Vallcorba, O., Rius, J., Frontera, C., Miravitlles, C. (2012) J. Appl. Cryst, 45, 1270-1277
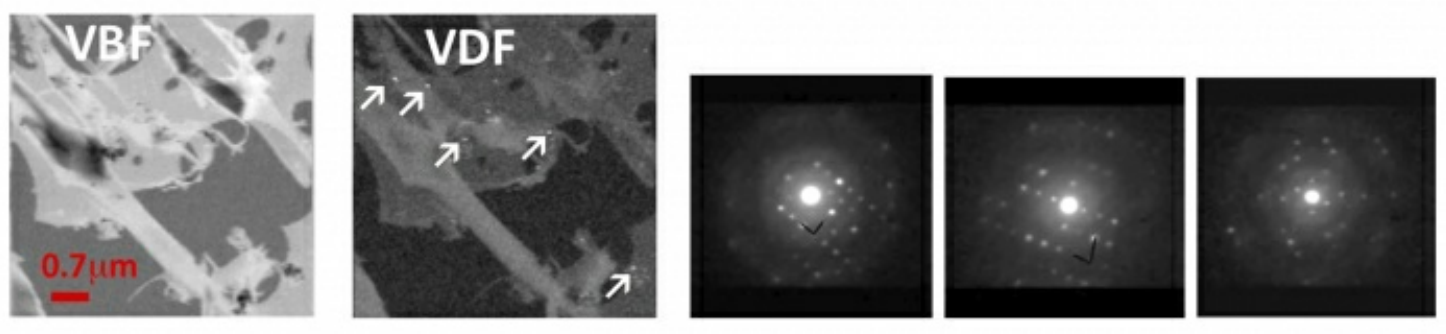

Figure (left) VBF (virtual bright field) and VDF (virtual dark field) maps where very small (10 nm) resorcinol crystals appear (white arrows) in amorphous $\mathrm{C}$ background (right) randomly selected ED patterns from aspirin crystals that have been used to determine unit cell and crystal structure

Keywords: Precession Electron Diffraction, Diffraction Tomography, pharmaceuticals 